\title{
Dynamic correlations in double-layer electron systems
}

\author{
B. Tanatar ${ }^{1}$ and B. Davoudi ${ }^{2}$ \\ ${ }^{1}$ Department of Physics, Bilkent University, Bilkent, Ankara 06533, Turkey \\ ${ }^{2}$ Institute for Studies in Theoretical Physics and Mathematics, Tehran 19395-5531, Iran
}

(Received 11 June 2000; published 6 April 2001)

\begin{abstract}
We study the effects of dynamic correlations on the ground-state properties of a double-layer twodimensional electron gas within the quantum Singwi-Tosi-Land-Sjölander theory (STLS). The intralayer and interlayer static structure factors, the pair-correlation functions, and the wave vector and frequency-dependent local-field factors have been calculated for a range of electron densities and layer separations. We find that the local-field factors have an oscillatory frequency dependence and the magnitude of interlayer local-field factors is about an order of magnitude smaller than that of the intralayer. Our results are compared with the randomphase approximation and the static STLS approximation to assess the importance of dynamical correlations. We also calculate the dispersion relations for the optical and acoustic plasmons and the damping of these modes to compare them with other mean-field theories, and we comment on the relevance of our results to the recent experiments.
\end{abstract}

DOI: 10.1103/PhysRevB.63.165328

PACS number(s): 73.21.-b, 73.20.Mf, 71.45.Gm

\section{INTRODUCTION}

The double-layer electron gas is a useful model to study various interesting phenomena arising from the interlayer interaction effects. It also provides the framework with which to study theoretically the experiments performed on double quantum-well structures made of semiconducting materials. ${ }^{1}$ In particular, when the density of electrons in the layers is low, the exchange and correlation effects give rise to a host of interesting many-body effects that are probed in experiments with and without external magnetic field. ${ }^{2}$

Advances in the past decade in growth and manufacturing techniques in semiconductor structures allowed for controlled experiments on these systems by changing the electronic density and layer separation. Our motivation for the present work comes from several recent experiments ${ }^{3-6}$ on double quantum-well structures in which dynamical correlation effects are thought to play an important role. In the Raman scattering experiments, ${ }^{3-5}$ the dispersion and damping of the plasmon modes are directly observed. The Coulomb drag measurements ${ }^{6}$ assess the contribution of plasmons indirectly through the temperature dependence of interlayer resistivity. In the analysis of these experimental results, it is stated that the dynamic correlation effects must be invoked to explain the observed discrepancies between the existing theories. Our calculations should be useful as an attempt to understand the dynamic correlation effects and particularly the damping properties of the plasmon modes in these systems.

The long-range Coulomb interaction is responsible for many of the interesting effects, such as the magnetic and crystalline ordering in single-layer electron systems. In a double-layer system, the enhanced correlation effects along with the additional degree of freedom (i.e., the interlayer distance) should give rise to an even richer phase diagram. ${ }^{7}$ The random-phase approximation (RPA) (Ref. 8) has been very successful in explaining such one-electron properties as the self-energy and quasiparticle lifetime and to a certain degree the collective modes, but it fails to provide a physi- cally acceptable pair-correlation function and correlation energy. The exchange and correlation effects not accounted for within the RPA are improved in the self-consistent-field approach of Singwi, Tosi, Land, and Sjölander ${ }^{9}$ (STLS). There have been many works utilizing the STLS method to study the correlation effects in double-layer electron-electron ${ }^{10-13}$ and electron-hole systems. ${ }^{14}$ Correlation effects in coupled electron and hole layers were also considered by the more sophisticated hypernetted-chain scheme. ${ }^{15}$

The dynamical correlation effects usually embodied in the frequency dependence of the local-field factor have been considered in a number of works using a variety of methods. Devreese, Brosens, and Lemmens, ${ }^{16}$ Awa, Yasuhara, and Asahi, ${ }^{17}$ and Singwi and co-workers ${ }^{18}$ have studied the dynamic properties of an electron gas using the diagrammatic perturbation theory. In a series of papers, Green and collaborators ${ }^{19}$ have studied the electron-gas problem within a conserving approximation. Takayanagi and Lipparini ${ }^{20}$ have solved Dyson's equation in the particle-hole channel to determine the effective interaction between electrons, and they have calculated the dynamic dielectric function. Nakano and Ichimaru ${ }^{21}$ used the Green's-function formalism and dynamical version of the convolution approximation to calculate various ground-state properties. A different approach to calculate the dynamic local-field factor was put forward by Hasegawa and Shimuzu, ${ }^{22}$ which replaces the original STLS scheme by a quantum-mechanical framework involving Wigner distribution functions (to be referred to as qSTLS). Holas and Rahman, ${ }^{23}$ Schweng and Böhm, ${ }^{24}$ Moudgil et $a l .{ }^{25}$ and Bulutay and Tanatar ${ }^{26}$ have employed the qSTLS formalism to study various aspects of dynamical correlations in homogeneous electron liquids. In the qSTLS scheme, the dynamics of the Pauli correlation hole makes the local-field corrections frequency-dependent, and the Coulomb correlation hole is not included. The overall picture that emerges from these applications is that the qSTLS scheme not only modifies such dynamic quantities as the plasmon dispersion and the dynamic structure factor, but the static correlation functions such as the pair distribution function and static 
susceptibility are also affected by the dynamic correlations. As the experimental efforts have been advancing in recent years, the prospects of detecting the many-body effects and being able to distinguish between different theories appear to be possible.

In this work, we extend the formalism of the dynamic STLS approach to double-layer systems and provide numerical calculations for the dynamic local-field corrections. Our aim is to first show the feasibility of such calculations and then make some detailed comparisons with other theoretical works that consider only the static correlations. As the dynamical effects have been becoming noticeable in recent experiments and several theoretical considerations, ${ }^{27,28}$ our study of the frequency dependence of the local-field factors should be useful in understanding various related phenomena.

The rest of this paper is organized as follows. In the next section, we first outline the model of a double-layer electrongas system and introduce the formalism of the qSTLS approach. In Sec. III, we present and discuss our results for the static and dynamical properties of a double-layer electron system. We conclude in Sec. IV with a brief summary and outlook to future work.

\section{THEORY}

We consider two parallel layers of two-dimensional (2D) electron gas interacting via the long-range Coulomb potential, in the presence of a rigid positive background for charge neutrality. Neglecting the finite widths of quantum wells, the contribution of the higher subbands, and tunneling effects between the layers, the Coulomb interactions (in Fourier space) between electrons within the same layer and between different layers are given by $V_{11}(q)=2 \pi e^{2} / \epsilon_{0} q$ and $V_{12}(q)=\left(2 \pi e^{2} / \epsilon_{0} q\right) e^{-q d}$, respectively, where $d$ is the interlayer distance and $\epsilon_{0}$ is the background dielectric constant. We neglect disorder effects since the experimental systems are typically very clean (i.e., of high mobility). We further assume that the density of electrons on both of the layers is the same, in which case the system is characterized by the dimensionless density parameter $r_{s}=1 / \sqrt{\pi n a_{B}^{*}}$, where $a_{B}^{*}$ $=\hbar^{2} \epsilon_{0} / e^{2} m^{*}$ is the effective Bohr radius defined in terms of the dielectric constant $\epsilon_{0}$ and electron effective mass $m^{*}$. In the numerical calculations below, we shall specialize to the GaAs systems for which experiments are performed.

The static STLS approximation improves upon the RPA by considering the local depletion of the electron density around any given particle. The short-range correlation effects neglected by the RPA are described by static local-field factors $G_{i j}(q)$, which follow from the assumption that the twoparticle distribution function may be decoupled as a product of two one-particle distribution functions multiplied by the pair-correlation function. In the qSTLS scheme, a similar idea is used, except that classical distribution functions are replaced by Wigner functions. The equations of motion for the Wigner distribution functions in a double-layer system, coupled with the linear-response theory, yield in the qSTLS approach the following expression for the density-density response functions:

$$
\begin{array}{r}
\chi_{i j}(q, \omega) \\
\quad=\frac{\chi_{0}(q, \omega)\left\{\delta_{i, j}-\eta_{i, j} V_{i j}(q)\left[1-G_{i j}(q, \omega)\right] \chi_{0}(q, \omega)\right\}}{\Delta(q, \omega)},
\end{array}
$$

where $i, j=1,2$ are the layer indices, $\eta_{i, j}=(-1)^{\delta_{i, j}}$, and $\Delta(q, \omega)$ is the dielectric function for the double-layer system given by

$$
\begin{aligned}
\Delta(q, \omega)= & \left\{1-\chi_{0}(q, \omega) V_{11}(q)\left[1-G_{11}(q, \omega)\right]\right\}^{2} \\
& -\left\{\chi_{0}(q, \omega) V_{12}(q)\left[1-G_{12}(q, \omega)\right]\right\}^{2} .
\end{aligned}
$$

In the above expressions, $\chi_{0}(q, \omega)$ is free-electron response function and $G_{11}(q, \omega)$ and $G_{12}(q, \omega)$ are the intralayer and interlayer dynamic local-field factors, respectively. The real and imaginary parts of the noninteracting response function are given by

$$
\begin{gathered}
\chi_{0}^{\prime}(q, \omega)=-\frac{k_{F}}{\pi q}\left\{q / k_{F}+\operatorname{sgn}\left(\nu_{+}\right) \theta\left(\nu_{+}^{2}-1\right)\left(\nu_{+}^{2}-1\right)^{1 / 2}\right. \\
\left.+\operatorname{sgn}\left(\nu_{-}\right) \theta\left(\nu_{-}^{2}-1\right)\left(\nu_{-}^{2}-1\right)^{1 / 2}\right\}, \\
\chi_{0}^{\prime \prime}(q, \omega)=-\frac{k_{F}}{\pi q}\left\{\theta\left(1-\nu_{+}^{2}\right)\left(1-\nu_{+}^{2}\right)^{1 / 2}\right. \\
\left.-\theta\left(1-\nu_{-}^{2}\right)\left(1-\nu_{-}^{2}\right)^{1 / 2}\right\},
\end{gathered}
$$

where $\operatorname{sgn}(x)=x /|x|, \theta(x)$ is the unit step function, and $\nu_{ \pm}=\left[ \pm \omega /\left(k_{F} q\right)-q /\left(2 k_{F}\right)\right]$.

The decoupling scheme in qSTLS theory leads to the following expressions for the dynamic local-field factors:

$$
\begin{aligned}
G_{i j}(q, \omega)= & -\frac{1}{n} \int \frac{d^{2} k}{(2 \pi)^{2}} \frac{\chi_{0}(\mathbf{q}, u, \omega)}{\chi_{0}(q, \omega)} \\
& \times \frac{q}{k}\left[S_{i j}(|\mathbf{q}-\mathbf{k}|)-\delta_{i, j}\right] \exp \left(-(k-q) z_{i j}\right),
\end{aligned}
$$

where $S_{i j}(q)$ are the partial static structure factors, $\chi_{0}(\mathbf{q}, u, \omega)$ is the inhomogeneous free-electron response function, ${ }^{29}$ and $z_{i j}=\left(1-\delta_{i, j}\right) d$. The real and imaginary parts of the inhomogeneous free response function are expressed as

$$
\begin{aligned}
\chi_{0}^{\prime}(q, u, \omega)= & -\frac{k_{F}}{\pi q}\left\{u /\left(q^{*} k_{F}\right)+\operatorname{sgn}\left(\mu_{+}\right)\right. \\
& \times \theta\left(\mu_{+}^{2}-1\right)\left(\mu_{+}^{2}-1\right)^{1 / 2}+\operatorname{sgn}\left(\mu_{-}\right) \\
& \left.\times \theta\left(\mu_{-}^{2}-1\right)\left(\mu_{-}^{2}-1\right)^{1 / 2}\right\}, \\
\chi_{0}^{\prime \prime}(q, u, \omega)= & -\frac{k_{F}}{\pi q}\left\{\theta\left(1-\mu_{+}^{2}\right)\left(1-\mu_{+}^{2}\right)^{1 / 2}\right. \\
& \left.-\theta\left(1-\mu_{-}^{2}\right)\left(1-\mu_{-}^{2}\right)^{1 / 2}\right\},
\end{aligned}
$$


where $\mu_{ \pm}=\left[ \pm \omega /\left(k_{F} q\right)-u /\left(2 q k_{F}\right)\right]$ and $u=\mathbf{q} \cdot \mathbf{k}$. Finally, the static structure factors are related to response functions via the fluctuation-dissipation theorem

$$
S_{i j}(q)=-\frac{1}{n \pi} \int d \omega \chi_{i j}^{\prime \prime}(q, \omega),
$$

which completes the set of self-consistent equations [i.e., Eqs. (1), (4), and (6)] to be solved iteratively to obtain the ground-state properties.

The above Eqs. (1), (4), and (6) along with Eqs. (3) and (5) can be solved self-consistently to obtain the dynamic local-field factors and static structure factors. With these functions at our disposal, we obtain other physical quantities such as the intralayer and interlayer pair-correlation functions, collective excitation energies, and their damping. The pair-correlation functions are related to static structure factors via the Fourier transform

$$
g_{i j}(r)=1+n \int \frac{d^{2} q}{(2 \pi)^{2}}\left[S_{i j}(q)-\delta_{i, j}\right] \exp (i \mathbf{q} \cdot \mathbf{r}),
$$

and the plasmon dispersion relations and their damping can be obtained by solving the equation $\Delta(q, \omega)=0$. In the actual computations, we have used functions evaluated on the imaginary frequency axis [i.e., $\chi_{0}(q, i \omega)$ and $\left.G(q, i \omega)\right]$, followed by analytic continuation $i \omega \rightarrow \omega+i \eta$, to find the physical quantities. This procedure facilitates the numerical work involved because all the functions involved in this case are real and their dependence on $\omega$ is very smooth. Finally, we mention that the static STLS equations may be recovered by expanding the Fermi distribution functions $f\left(k \pm \hbar q^{\prime} / 2\right)$ appearing in the inhomogeneous response function $\chi_{0}\left(q, q^{\prime} ; \omega\right)$ in Taylor series and then taking the limit $\hbar$ $\rightarrow 0$. The RPA results are obtained, say, in the dynamic susceptibility expressions by setting $G_{i j}(q, \omega)=0$.

\section{NUMERICAL RESULTS}

In this section, we present the numerical result of our calculations for the static and dynamical properties of a double-layer electron system, which have been obtained in the qSTLS approximation. We assume that the electron density is the same for both layers and we display results for various densities (expressed in terms of the dimensionless parameter $r_{s}$ ) and layer separations. For the most part, we shall compare our results with those from the static STLS calculations to make a ready assessment of the effects of dynamic correlations. We use material parameters appropriate for GaAs structures where the dielectric constant is $\epsilon_{0}$ $\approx 12.9$ and the electron effective mass is $m^{*}=0.07 m$.

\section{A. Correlation functions}

We first display in Fig. 1(a) the intralayer and interlayer static structure factors $S_{i j}(q)$ for $r_{s}=4$ and for $d=200 \AA$. The existence of a peak around $q=3.5 k_{F}$ for $S_{11}(q)$ in the qSTLS approximation shows that the correlation effects are better accounted for in this approach compared to the static STLS and RPA approximations. We show in Fig. 1(b) the
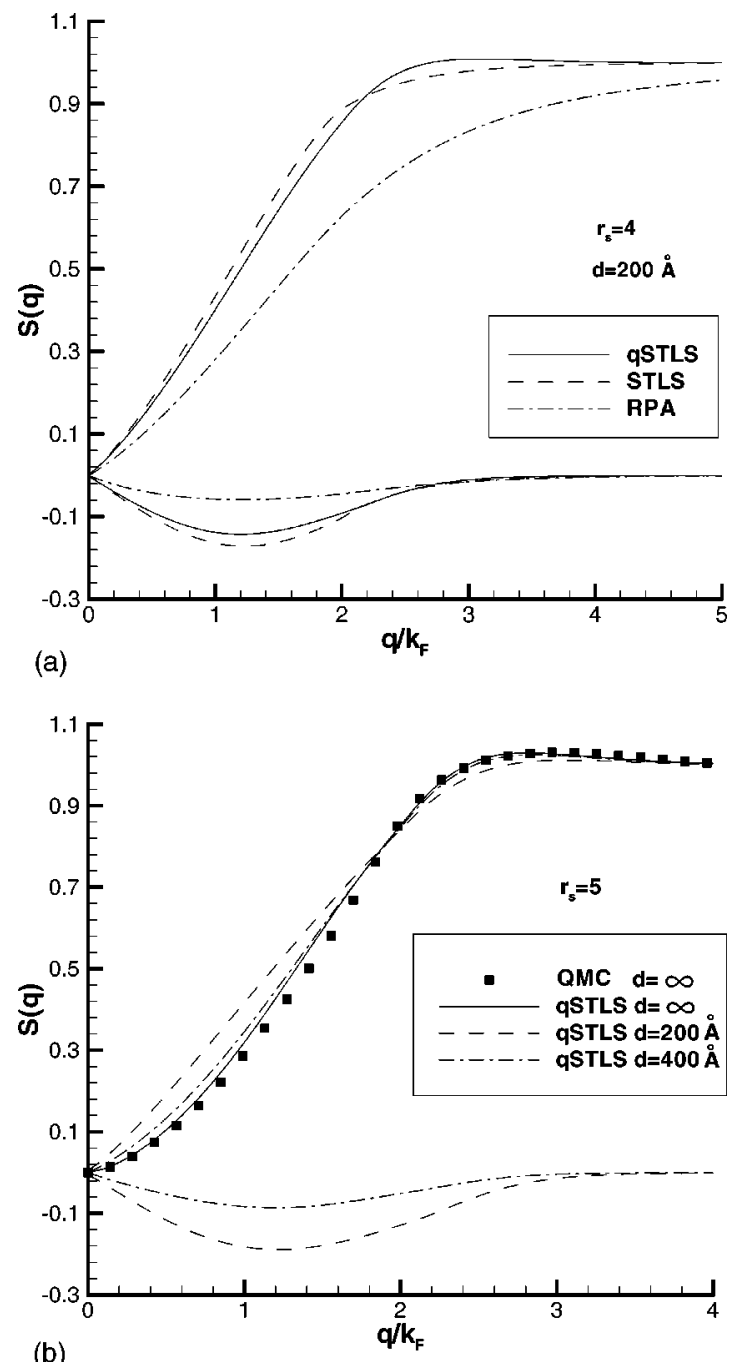

FIG. 1. (a) The intralayer and interlayer static structure factors $S_{11}(q)$ (upper curves) and $S_{12}(q)$ (lower curves) for $r_{s}=4$ and $d$ $=200 \AA$. The solid, dashed, and dash-dotted lines correspond to the results of the qSTLS, the STLS, and the RPA approximations, respectively. (b) $S_{11}(q)$ and $S_{12}(q)$ at $r_{s}=5$ in the qSTLS for different values of $d$. Solid squares are QMC results for a single layer from Ref. 10.

intralayer and interlayer static structure factors $S_{i j}(q)$ for $r_{s}$ $=5$, at different layer separations $d=200 \AA$ and $d=400 \AA$. We observe that, as the distance between the layers is increased, the interlayer structure factor $S_{12}(q)$ tends to diminish and the intralayer structure factor $S_{11}(q)$ approaches that of a single-layer system, as expected. Also shown in the same figure is the result of a single-layer structure factor calculated within the quantum Monte Carlo (QMC) simulation. ${ }^{30}$ It is clear from Fig. 1(b) that there is good agreement between the qSTLS and the QMC results at this density, which lends credence to the scheme incorporating dynamic correlation effects. We also observe that the height of the peak in $S_{11}(q)$ becomes slightly larger as the distance between the layers increases. QMC simulations for doublelayer electron systems were also performed, ${ }^{31}$ but we are not able to make a direct comparison since the static structure factors were not reported. It is interesting to note that our 


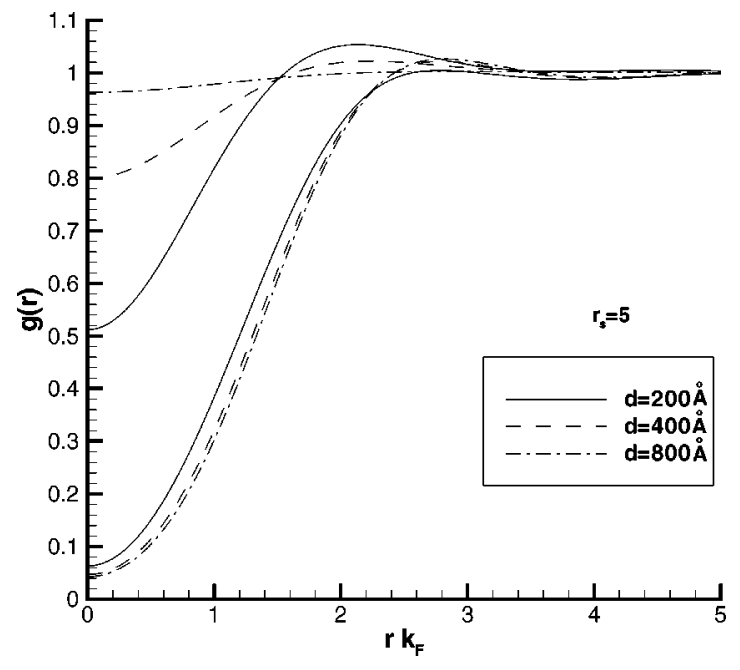

FIG. 2. The intralayer (lower curves) and interlayer (upper curves) pair-correlation functions $g_{11}(r)$ and $g_{12}(r)$ at $r_{s}=5$ and different values of $d$.

$T=0$ calculations show that $S_{11}(q=0)=S_{12}(q=0)=0$, which has been obtained in many other works on doublelayer systems. The first part of the equality basically follows from the perfect screening sum rule. The limiting forms are further corroborated by the RPA results, which should be valid in the long-wavelength limit. In the case of classical systems, because of the relation $S_{i j}(q) \sim \chi_{i j}(q)$, where $\chi_{i j}(q)$ is the static susceptibility, one typically finds $S_{11}(q$ $=0)=S_{12}(q=0) \neq 0$.

The Fourier transform of the static structure factors $S_{i j}(q)$ yields the partial pair-correlation functions $g_{i j}(r)$. The results for intralayer and interlayer pair-correlation functions are depicted in Fig. 2 for $r_{s}=5$ at layer separation distances $d=200,400$, and $800 \AA$. We note that the interlayer paircorrelation function is affected by the changes in the layer separation more dramatically than the intralayer paircorrelation function. As $g_{12}(0)$ decreases with increasing $d$, a peak structure develops around $r \approx 2 k_{F}^{-1}$. There is also a slight peak in $g_{11}(r)$, which tends to increase with increasing $d$. These results are in qualitative agreement with the previous calculations involving static local-field factors. ${ }^{14}$

Our self-consistent calculations yield the static structure factors and local-field factors simultaneously for a given density and layer separation. Our calculations are done on the imaginary frequency axis for computational convenience. The dependence of the local-field factors on real frequencies is of major interest. To investigate the behavior of the localfield factors for real frequencies, we first define the following integral:

$$
\begin{aligned}
\psi_{i j}(q, \omega)= & -2 \int d k\left[S_{i j}(k)-1\right] \int_{q^{2}-q k}^{q^{2}+q k} d u \\
& \times \frac{\chi_{0}(q, u, \omega) \exp \left(-k_{F} z_{i j}\left(\sqrt{2 u+k^{2}-q^{2}}-q\right)\right)}{\sqrt{1-\left[\left(q^{2}-u\right) /(q k)\right]^{2}} \sqrt{2 u+k^{2}-q^{2}}},
\end{aligned}
$$
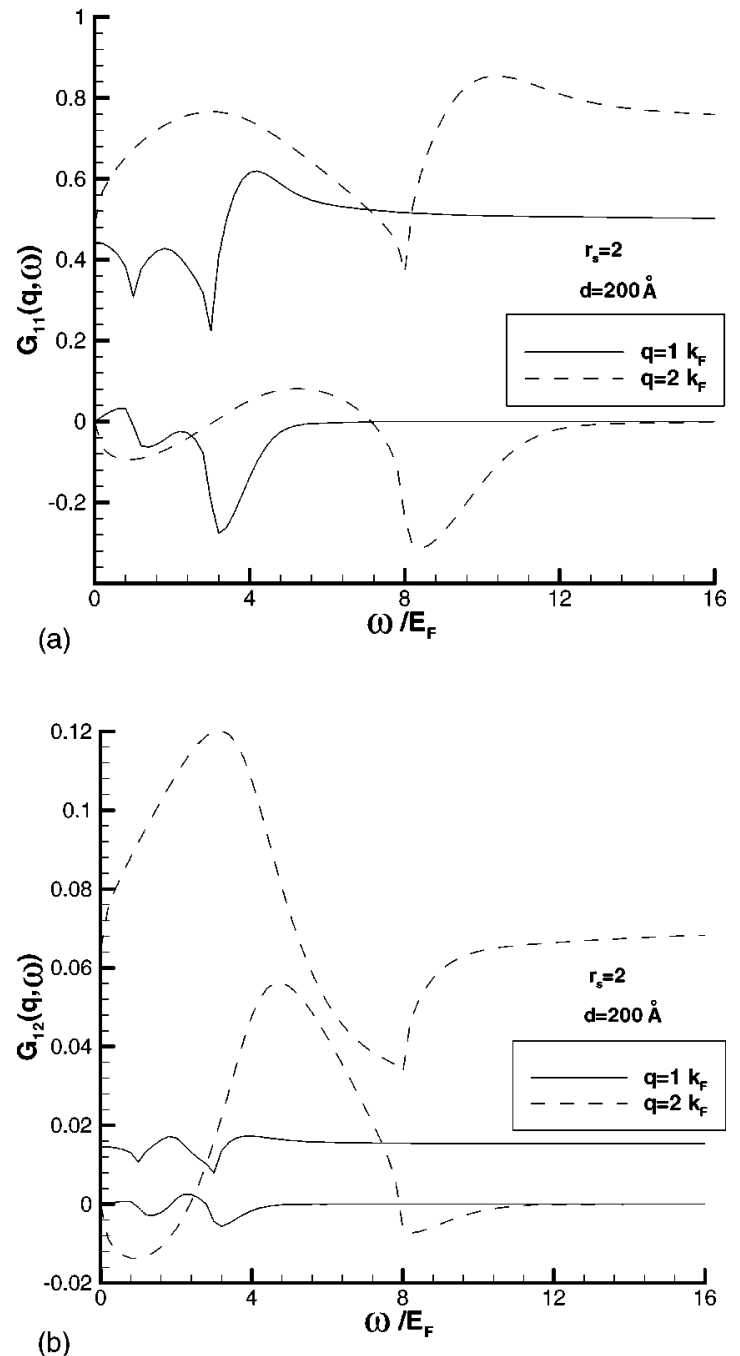

FIG. 3. The frequency dependence of the (a) intralayer and (b) interlayer local-field factors $G_{11}(q, \omega)$ and $G_{12}(q, \omega)$ at $r_{s}=2$ and $d=200 \AA$.

so that $G_{i j}(q, \omega)=\psi_{i j}(q, \omega) / \chi_{0}(q, \omega)$. In the calculation of $G_{i j}(q, \omega)$, we use the final converged static structure factors in Eq. (6). In Fig. 3(a), we show the real and imaginary parts of the intralayer $G_{11}(q, \omega)$ as a function of $\omega$ for two values of the wave vector. Both the real and imaginary parts exhibit oscillatory behavior, the details of which depend on $q$, as in the previous calculations. ${ }^{23-26}$ At large values of $\omega$, $G_{11}(q, \omega)$ tends to a constant. The imaginary part of $G_{11}(q, \omega)$ vanishes at $\omega=0$ as required by general causality relations. The frequency dependence of the interlayer localfield factor $G_{12}(q, \omega)$ is shown in Fig. 3(b). The qualitative features of $G_{12}(q, \omega)$ are similar to that of $G_{11}(q, \omega)$. The magnitude of $G_{12}(q, \omega)$ is about an order of magnitude smaller than that of its intralayer counterpart. This suggests that in most practical applications the frequency dependence of the interlayer local-field factors may be neglected and the static local-field factor may account for the interlayer correlation effects. However, in applications such as the Coulomb drag effect in which integrals over the wave vector and frequency are performed, their cumulative effect may be impor- 


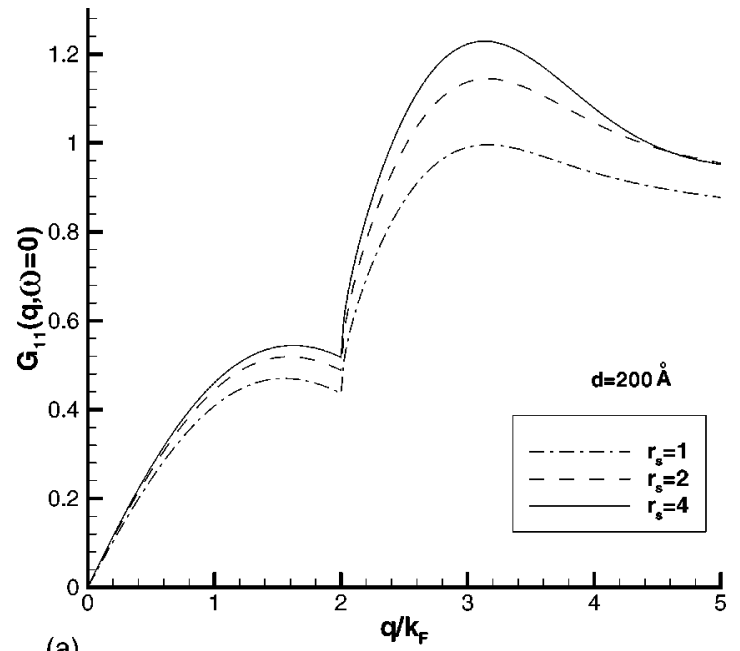

(a)

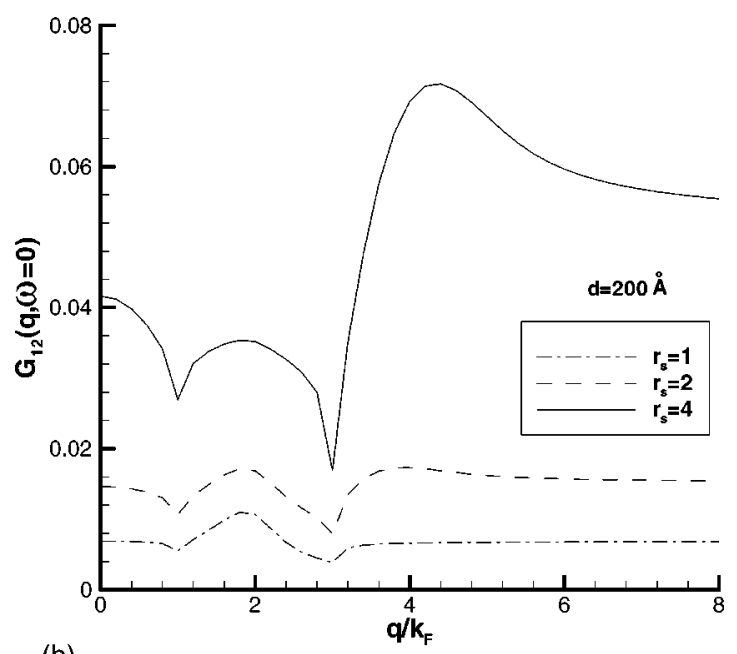

(b)

FIG. 4. The zero-frequency limit of the (a) intralayer and (b) interlayer dynamic local-field factors $G_{11}(q, 0)$ and $G_{12}(q, 0)$ at $d$ $=200 \AA$ and various $r_{s}$ values.

tant. As we shall discuss later, the imaginary parts of $G_{i j}(q, \omega)$ play an important role in the damping properties of plasmons. We mention in passing that once the full frequency-dependent local-field factors are obtained, one can construct the effective interactions $V_{i j}^{\text {eff }}=V_{i j}(q)[1$ $\left.-G_{i j}(q, \omega)\right]$ to study various phenomena such as Coulombinteraction-induced superconductivity. ${ }^{32}$

The zero and large frequency limits of the local-field factor are of interest, because most mean-field theories use frequency-independent, static $G(q)$. In Fig. 4(a), we display the zero-frequency sector of the self-consistently calculated intralayer local-field factor $G_{11}(q, 0)$ as a function of $q$, for several densities. $G_{11}(q, 0)$ exhibits features not encountered in theories yielding a static $G(q)$. First, there is a sharp point of inflection at $2 k_{F}$, and there is a maximum around $q$ $\approx 3 k_{F}$ that exceeds unity at large $r_{s}$. The behavior of the interlayer local-field factor at zero frequency $G_{12}(q, 0)$ is shown in Fig. 4(b). There appear to be two dips at $k_{F}$ and $3 k_{F}$ in the overall shape of $G_{12}(q, 0)$, and a peak developing around $q \approx 4 k_{F}$ at large $r_{s}$.
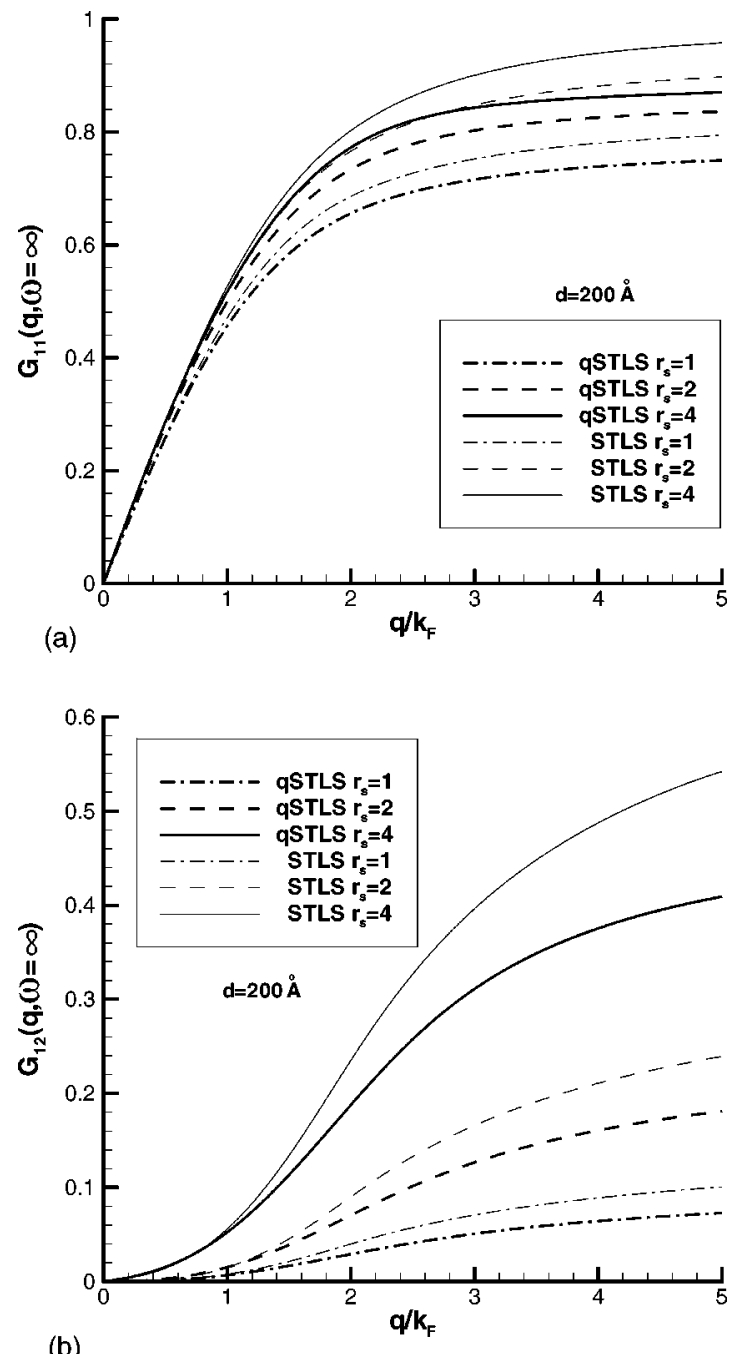

(b)

FIG. 5. The large frequency limit of the (a) intralayer and (b) interlayer dynamic local-field factors $G_{11}(q, \infty)$ and $G_{12}(q, \infty)$ at $d=200 \AA$ and various $r_{s}$ values. Also shown for comparison are the corresponding static STLS results.

It can be shown that for fixed $q$ and large $\omega$, the localfield factors behave as $\lim _{\omega \rightarrow \infty} G_{i j}(q, \omega)=G_{1 j}(q)$ $+O\left(1 / \omega^{2}\right)$, where $G_{i j}(q)$ are the static local-field factors of the STLS approximation. Their explicit forms are given by ${ }^{10-12}$

$$
\begin{aligned}
G_{i j}(q)= & -\int \frac{d^{2} k}{(2 \pi)} \frac{\mathbf{q} \cdot \mathbf{k}}{q k}\left[S_{i j}(|\mathbf{q}-\mathbf{k}|)-\delta_{i, j}\right] \\
& \times \exp \left(-k_{F}(k-q) z_{i j}\right) .
\end{aligned}
$$

We observe in Figs. 5(a) and 5(b) that the high-frequency limits $G_{i j}(q, \infty)$ approximate the static local-field factors $G_{i j}(q)$ better than $G_{i j}(q, 0)$. Particularly for small $q$ (i.e., $q$ $\left.\lesssim k_{F}\right), G_{i j}(q, \infty)$ and $G_{i j}(q)$ are very similar. At larger wave vectors, and with increasing $r_{s}$, however, $G_{i j}(q, \infty)$ and $G_{i j}(q)$ show notable differences, which we can attribute to the $O\left(1 / \omega^{2}\right)$ corrections, which are reflected in the different 


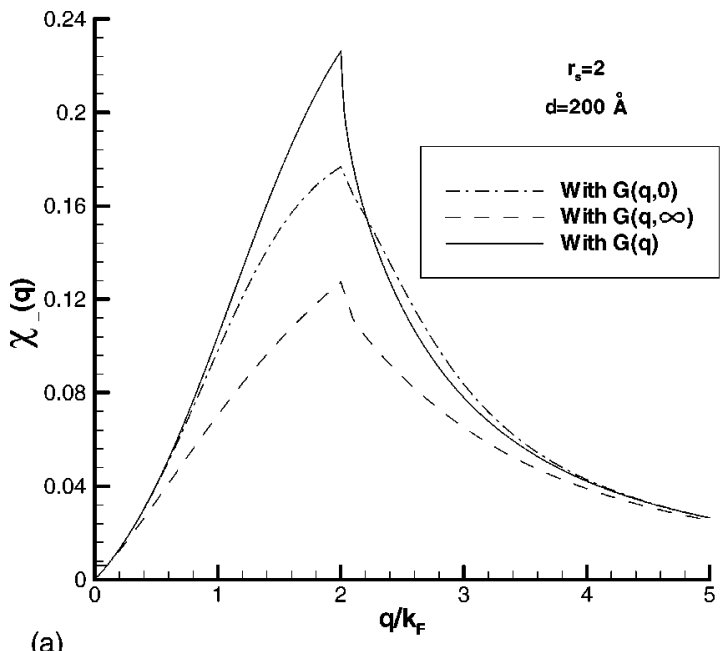

(a)

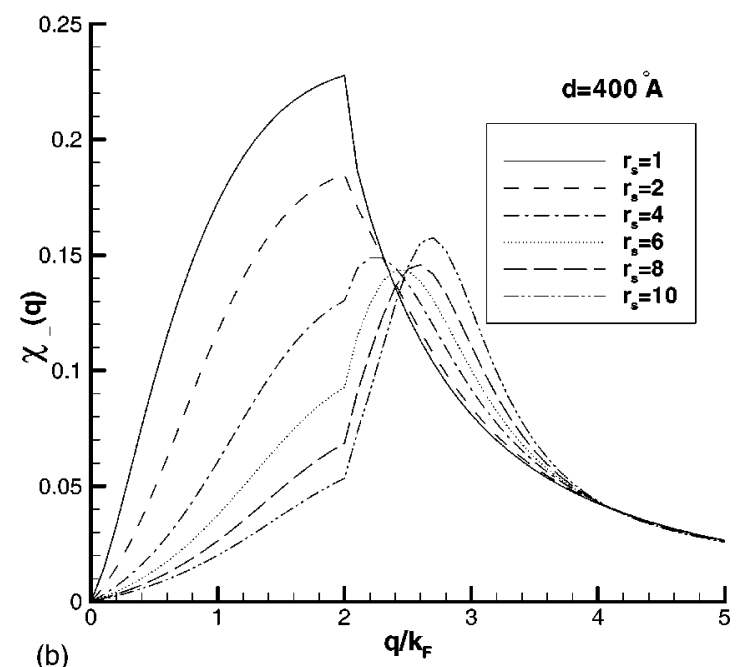

FIG. 6. (a) The out-of-phase static susceptibility $\chi_{-}(q)$ (in units of $m / k_{F}$ ) at $r_{s}=2$ and $d=200 \AA$ using various local-field factors. (b) $\chi_{-}(q)$ (in units of $m / k_{F}$ ) at $d=200 \AA$ for different densities, calculated using $G_{i j}(q, 0)$.

static structure factors entering Eq. (9). We observe that large $q$ limits of $G_{i j}(q, \infty)$ are consistently lower than those of $G_{i j}(q)$.

To see the effects of various local-field factors on the physical quantities, we calculate the static density-density response functions for the double-layer system. Defining the in-phase and out-of-phase static response functions as $\chi_{ \pm}(q)=\chi_{11}(q) \pm \chi_{12}(q)$, we show in Fig. 6(a), $\chi_{-}(q)$ at $r_{s}=2$ and $d=200 \AA$. As can be seen, the use of the zerofrequency $G(q, 0)$ and large-frequency $G(q, \infty)$ limits of the dynamic local-field factors and the static local-field factor $G(q)$ affect the static susceptibility considerably. These differences will bring quantitative changes to the critical parameters of the instability discussed by Liu et al. ${ }^{3}$ Strictly speaking, the zero-frequency limit $G_{i j}(q, 0)$ should be used in the static susceptibility calculations. In Fig. 6(b), we show $\chi_{-}(q)$ calculated using $G_{i j}(q, 0)$ at different densities for a layer separation of $d=400 \AA$. As $r_{s}$ increases, the shape of $G_{i j}(q, 0)$ shown in Figs. 4(a) and 4(b) becomes more influential, and we observe that the peak position of $\chi_{-}(q)$ shifts towards $q \approx 3 k_{F}$. The peak value of $\chi_{-}(q)$, after an initial decrease, starts to increase. This is similar to the chargedensity-wave instability developing at low density studied by Neilson et al. ${ }^{12}$ The perfect screening sum rule applied to a double-layer system states that $\chi_{11}(q)=-\chi_{12}(q)$ as $q \rightarrow 0$. Using the definitions of $\chi_{ \pm}(q)$ and the small $q$ behavior of $\chi_{i j}(q)$, we find that $\chi_{-}(q \rightarrow 0)=0$ and $\chi_{+}(q)$ approaches a constant. Our results for $\chi_{-}(q)$ in Figs. 6(a) and 6(b) and $\chi_{+}(q)$ (not shown) verify this expected behavior.

\section{B. Plasmon dispersion and damping}

The collective modes of double-layer electron systems were first treated within the RPA. ${ }^{33}$ The charge-density fluctuations in the two layers that are in phase and out of phase are termed optical and acoustic plasmons, respectively. In the long-wavelength limit and within the RPA, these plasmons behave as

$$
\begin{gathered}
\omega_{\mathrm{op}}(q) \simeq \sqrt{q v_{F} k_{\mathrm{TF}}}, \\
\omega_{\mathrm{ap}}(q) \simeq q v_{F} \frac{1+d k_{\mathrm{TF}}}{\sqrt{1+2 v_{F} k_{\mathrm{TF}}}},
\end{gathered}
$$

where $k_{\mathrm{TF}}=2 / a_{B}^{*}$ is the Thomas-Fermi wave vector and $v_{F}$ is the Fermi velocity. The higher-energy mode with $\sim \sqrt{q}$ behavior is called the optical plasmon, whereas the lowerenergy mode with $\sim q$ behavior is called the acoustic plasmon. Within the RPA, the plasmon modes are undamped when they are outside the particle-hole excitation region. We note that the underlying collective modes in the RPA and STLS approximation have been criticized within the quasilocalized charge approximation scheme, ${ }^{34}$ which predicts a gap in the out-of-phase mode instead of a linear (acoustic) dispersion. However, such a gap forms at a much larger value of $r_{s}$, perhaps close to the crystallization density. Such a discrepancy between the qSTLS and the quasilocalized charge approximation may be due to the role played by the third-moment sum rule, which we discuss below. On the other hand, it has been argued by Ortner ${ }^{35}$ that in this approach the neglect of damping processes overestimates the role of correlations and the correct account of damping restores the situation to yield an acoustic plasmon mode without a gap. In view of the contradicting nature of the various predictions, it would be interesting to further study the collective modes in double-layer systems experimentally, especially in the $q \rightarrow 0$ limit.

We show the optical and the acoustic plasmon dispersions within the qSTLS approximation for $r_{s}=4$ and several layer separation distances in Fig. 7(a). The density dependence of the plasmon modes is illustrated in Fig. 7(b). For a fixed density, the optical and acoustic plasmons are pushed apart with decreasing layer separation. The acoustic plasmons enter the single-particle excitation region at a smaller wave vector. At even smaller layer separations, the acoustic plasmons cease to exist as well-defined excitations and lie entirely within the single-particle excitation region. Much of these results are qualitatively similar to those found in the RPA and static STLS approximation. The main quantitative 


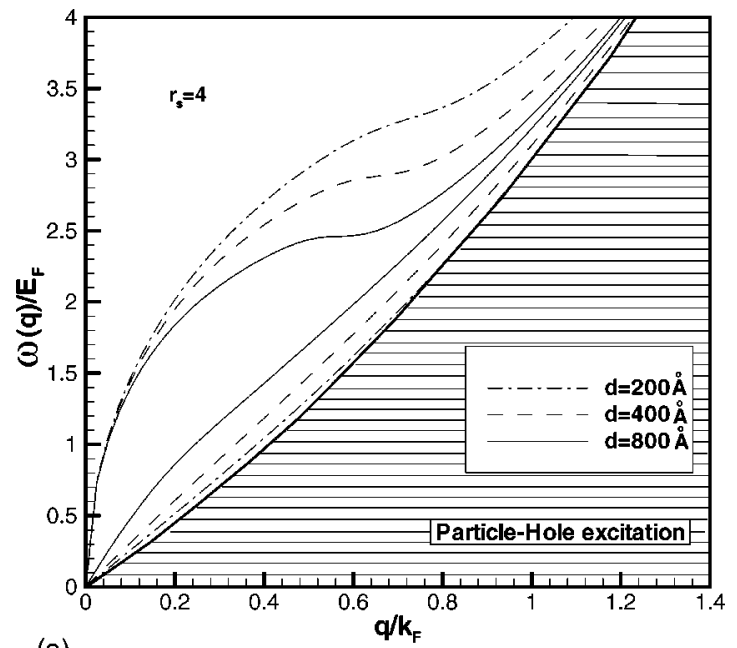

(a)

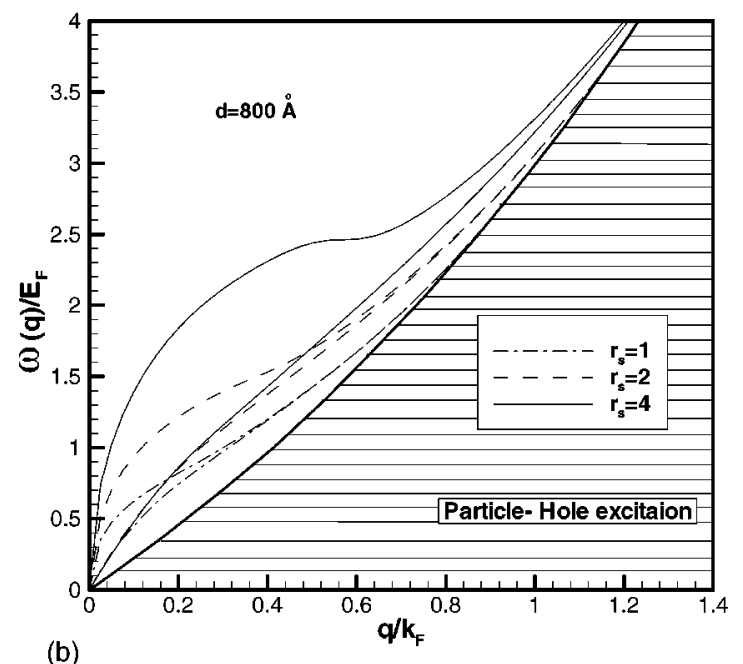

(b)

FIG. 7. (a) The dispersion of optical and acoustic plasmons at $r_{s}=4$ and $d=200 \AA, 400 \AA$, and $800 \AA$. (b) The density dependence of plasmon modes at $d=800 \AA$ and $r_{s}=1,2$, and 4 .

difference is that plasmon dispersions within the qSTLS typically lie between the RPA and static STLS results. Such a behavior was also encountered in single-layer ${ }^{25}$ and 1D electron systems. ${ }^{26}$ For a given layer density, there is a critical layer spacing below which the acoustic plasmon lies entirely within the single-particle excitation region. ${ }^{33}$ In the present context, this critical spacing is approximately given by $d_{c}=\lim _{q \rightarrow 0} G_{11}^{\prime}-G_{12}^{\prime}$, where the local-field factors are the large frequency limits $G_{i j}(q, \infty)$ and the primes denote differentiation with respect to $q$. We note that because $G_{i j}(q, \infty)$ lies below the static $G_{i j}(q)$, the value of $d_{c}$ will in general be lowered in the qSTLS approximation.
The dispersion relation and the damping for plasmon modes in the present scheme are obtained from the zeros of the dielectric function $\Delta\left(q, \omega_{q}-i \gamma_{q}\right)=0$, where $\gamma_{q}$ represent the damping. We rewrite the dielectric function as

$\Delta\left(q, \omega_{q}-i \gamma_{q}\right)=\left[1-\phi_{11}\left(q, \omega_{q}-i \gamma_{q}\right)\right]^{2}-\phi_{12}\left(q, \omega_{q}-i \gamma_{q}\right)^{2}$,

where $\phi_{i j}$ are given by

$$
\phi_{i j}(q, \omega)=V_{i j}(q)\left[\chi_{0}(q, \omega)-\psi_{i j}(q, \omega)\right] .
$$

To find the roots of $\Delta$, we separate the real and imaginary parts

$$
\begin{gathered}
\Delta^{\prime}=\left(1-\phi_{11}^{\prime}\right)^{2}-\left(\phi_{12}^{\prime}\right)^{2}-\left(\phi_{11}^{\prime \prime}\right)^{2}+\left(\phi_{12}^{\prime \prime}\right)^{2}, \\
\Delta^{\prime \prime}=2\left[\left(1-\phi_{11}^{\prime}\right) \phi_{11}^{\prime \prime}-\phi_{12}^{\prime} \phi_{12}^{\prime \prime}\right] .
\end{gathered}
$$

To find the solution of the $\Delta\left(q, \omega_{q}-i \gamma_{q}\right)=0$, we must find $\omega_{q}$ and $\gamma_{q}$ in a way that both the real and imaginary parts of the above equation are equal to zero. In the STLS and RPA approximations, $\psi^{\prime \prime}=0$ for all values of $\omega$ and $q$, and the $\chi_{0}^{\prime \prime}(q, \omega)=0$ in the region, $\omega>q^{2}+2 q$. This means that the $\phi^{\prime \prime}=0$ or $\Delta^{\prime \prime}=0$ in the above region leading to undamped plasmon excitations. Within these approximations, we just need to find the solutions of the following equation:

$$
\begin{aligned}
\Delta^{\prime} & =\left(1-\phi_{11}^{\prime}\right)^{2}-\left(\phi_{12}^{\prime}\right)^{2} \\
& =\left[\left(1-\phi_{11}^{\prime}\right)-\phi_{12}^{\prime}\right]\left[\left(1-\phi_{11}^{\prime}\right)+\phi_{12}^{\prime}\right]=0 .
\end{aligned}
$$

In the qSTLS approximation, the frequency dependence of the local-field factors changes the situation, since $\phi_{i j}^{\prime \prime} \neq 0$ in any region. However, $\psi_{i j}^{\prime \prime}$ are typically small, hence the damping is not very large. For small damping, which is equivalent to small $\psi_{i j}^{\prime \prime}$, we can expand the dielectric function in terms of $\gamma_{q}$ and retain the most important terms. This means that we can neglect terms like $\gamma_{q} \psi^{\prime \prime}, \psi^{\prime \prime 2}$ and higherorder terms. Our analysis for $\omega_{q}$ and $\gamma_{q}$ is now based on the solution of the following equations:

$$
\begin{aligned}
& {\left[1-\phi_{11}^{\prime}\left(q, \omega_{q}^{\mathrm{op}}\right)\right]-\phi_{12}^{\prime}\left(q, \omega_{q}^{\mathrm{op}}\right)=0} \\
& {\left[1-\phi_{11}^{\prime}\left(q, \omega_{q}^{\mathrm{ap}}\right)\right]+\phi_{12}^{\prime}\left(q, \omega_{q}^{\mathrm{ap}}\right)=0}
\end{aligned}
$$

and more explicitly for the damping of the plasmon modes

$$
\gamma_{q}^{\mathrm{op}, \mathrm{ap}}=\left.\left|\frac{\left[1-\phi_{11}^{\prime}(q, \omega)\right] \phi_{11}^{\prime \prime}(q, \omega)-\phi_{12}^{\prime}(q, \omega) \phi_{12}^{\prime \prime}(q, \omega)}{\left[1-\phi_{11}^{\prime}(q, \omega)\right] \frac{d \phi_{11}^{\prime}(q, \omega)}{d \omega}-\phi_{12}^{\prime}(q, \omega) \frac{d \phi_{12}^{\prime}(q, \omega)}{d \omega}}\right|\right|_{\omega=\omega_{q}^{\mathrm{op}, a p}}
$$



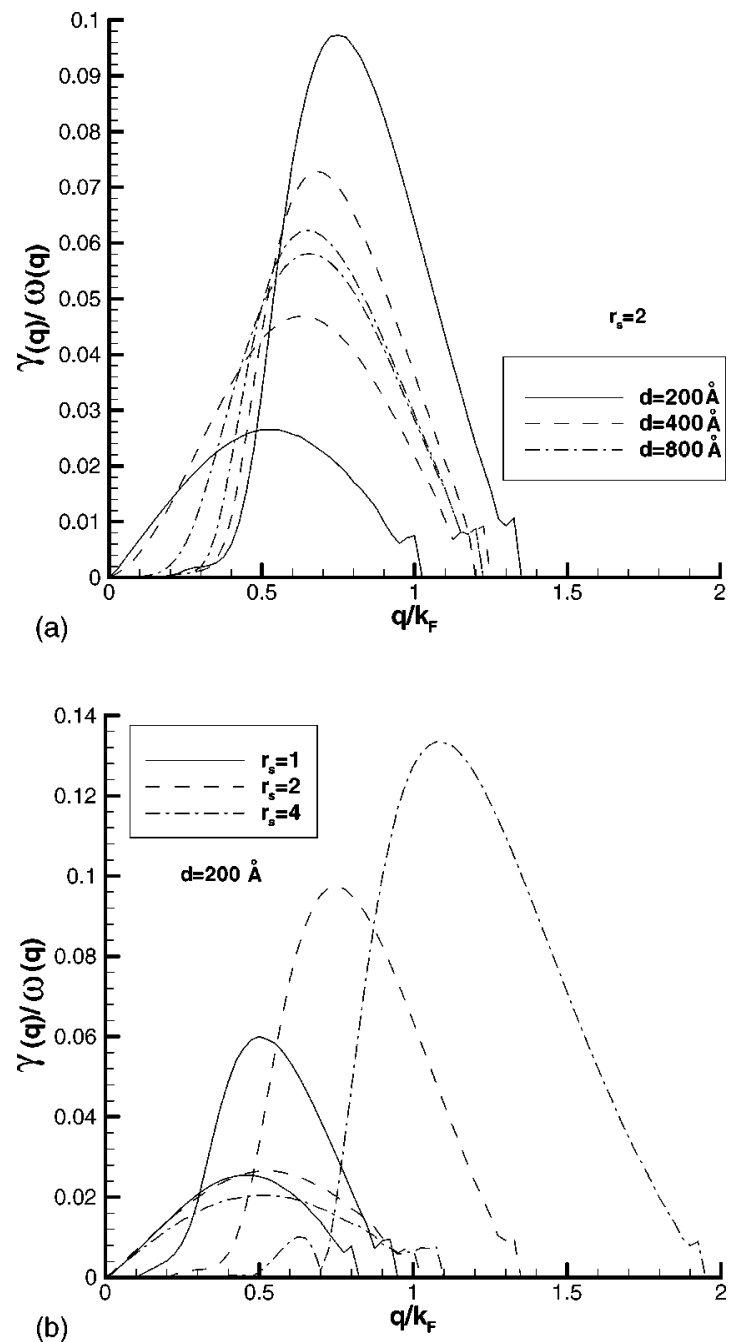

FIG. 8. (a) The damping of the optical and acoustic plasmons at $r_{s}=2$ and layer separations $d=200 \AA, 400 \AA$, and $800 \AA$. (b) The density dependence of the plasmon damping at $d=200 \AA$ and $r_{s}$ $=1,2$, and 4 .

We depict in Fig. 8(a) the damping in the optical (lower curves) and acoustic (upper curves) plasmon dispersions at $r_{s}=2$ and varying layer-separation distances. We observe that the peak values of acoustic plasmon damping is larger than that of the optical plasmon, the modes are damped outside the single-particle excitation region, and as the layer separation increases, the $\gamma_{q}$ for both of the modes approach each other. All of these features are in qualitative agreement with the experimental findings. In Fig. 8(b), we explore the dependence of $\gamma_{q}$ for optical and acoustic plasmons at different densities. At fixed layer separation $(d=200 \AA)$, as we decrease the density of electrons in each layer, the damping of the modes increases in magnitude and in the range of $q$ values. The recent experiments of Bhatti et al. ${ }^{4}$ and Kainth et $a l .{ }^{5}$ on double quantum-well systems were performed at finite temperature and a systematic study of the damping of acoustic plasmon was presented. We cannot confront the experiments since our calculations were carried out at $T=0$. The temperature dependence of the dynamic local-field fac- tors is largely unexplored in the literature. However, if we assume that $G_{i j}(q, \omega)$ depend weakly on $T$, our results of Figs. 8(a) and 8(b) will be qualitatively broadened by the temperature effects coming from the $T$ dependence of $\chi_{0}(q, \omega)$. Although further detailed work needs to be done in this direction, it is clear that an approach taking the dynamic correlations into account might be useful in understanding the experimental results. We also note that damping of the plasmon modes may also arise from coupling to multiparticle excitations, which are not considered in the present framework. The calculated values of $\gamma_{q} / \omega_{q}$ for a single-layer electron system ${ }^{36}$ are smaller than $0.5 \%$, which suggests that they are not as important as the damping brought about by the dynamical correlations.

A major shortcoming shared by the static STLS and the dynamic qSTLS approaches is that the third-frequency moment sum rule is not exactly satisfied. The reason for this is that the exact kinetic energy and the Pathak-Vashishta ${ }^{37}$ form of the local-field factor are replaced by the free-particle kinetic energy and $G_{\text {STLS }}$, respectively, in the present approximation. More precisely, in the third-frequency sum rule, the high-frequency local-field factors $G_{i j}(q, \infty)$ enter the expression. As the intermediate and large $q$ behavior of the plasmon dispersions is affected by the sum-rule requirements, our results are expected to change quantitatively but not qualitatively. It might be possible to construct a dynamical theory for the local-field factors along the lines discussed by Green et al. ${ }^{19}$ or Mukhopadhyay. ${ }^{38}$ The schemes introduced in these works, however, are more lengthy and require separate study.

\section{SUMMARY AND CONCLUDING REMARKS}

In this work, we have employed the self-consistent qSTLS scheme to calculate the dynamical intralayer and interlayer local-field factors describing the short-range correlation effects in a double-layer electron system. In the qSTLS theory, the dynamics of the Pauli correlation hole renders the local-field factors frequency-dependent. The dynamics of the Coulomb correlation hole is not included, but it is expected to be important at low electron densities. We have found that both the $G_{11}(q, \omega)$ and $G_{12}(q, \omega)$ exhibit oscillatory behavior as a function of frequency, and the magnitude of the interlayer local-field factor is typically an order of magnitude smaller than that of the intralayer counterpart. The dynamic local-field factors modify the plasmon dispersions such that both the optical and acoustic plasmons lie between the RPA and static STLS curves. Furthermore, the plasmon modes acquire damping in the present theory even outside the particle-hole excitation region where the mean-field theories with static local-field factors predict zero damping.

Our calculations can be improved upon and extended into several directions. As the qSTLS shares with STLS and RPA the same drawback of not fulfilling the compressibility sum rule, it may be possible to use the Vashishta-Singwi ${ }^{39}$ approach (rather its qSTLS version ${ }^{23}$ ) to obtain better agreement. Similarly, the third-frequency sum rule may be imposed by considering the quantum version of the Pathak-Vashishta ${ }^{37}$ scheme. Using the correlation functions 
obtained in this work, one can calculate the ground-state energy and other thermodynamic functions such as the pressure and compressibility. The results for these quantities are expected to be in good agreement with other calculations in the range $r_{s} \leqslant 5$. Experimentally, it is difficult to have matched densities in the double-layer system, and also having unequal densities gives rise to two particle-hole continua that would affect the collective mode structure. ${ }^{40}$ The formalism set out here can easily be extended to study this more involved case. A further direction to pursue would be to include the finitetemperature effects within the dynamical qSTLS scheme, as has been done for a 3D system. ${ }^{24}$ This would make the comparison with experiments measuring the temperature dependence of the damping of plasmons more meaningful. More- over, to make better contact with the experimental samples, the finite width of the electron layers may have to be incorporated in the calculations.

\section{ACKNOWLEDGMENTS}

The work of B.T. was partially supported by the Scientific and Technical Research Council of Turkey (TUBITAK) under Grant No. TBAG-2005, by NATO under Grant No. SfP971970, and by the Turkish Department of Defense under Grant No. KOBRA-001. B.D. acknowledges support from the Institute for Studies in Theoretical Physics and Mathematics, Tehran, Iran.
${ }^{1}$ See for recent reviews, L. Świerkowski, D. Neilson, and J. Szymański, Aust. J. Phys. 46, 423 (1993); C. B. Hanna, D. Haas, and J. C. Díaz-Vélez, Phys. Rev. B 61, 13882 (2000).

${ }^{2}$ See, for instance, Perspectives in Quantum Hall Effects, edited by S. Das Sarma and A. Pinczuk (Wiley, New York, 1997).

${ }^{3}$ A. Pinczuk, M. G. Lamont, and A. C. Gossard, Phys. Rev. Lett. 56, 2092 (1986); G. Fasol, N. Mestres, H. P. Hughes, A. Fischer, and K. Ploog, ibid. 56, 2517 (1986).

${ }^{4}$ A. S. Bhatti, D. Richards, H. P. Hughes, and D. A. Ritchie, Phys. Rev. B 53, 11016 (1996); D. S. Kainth, D. Richards, A. S. Bhatti, H. P. Hughes, M. Y. Simmons, E. H. Linfield, and D. A. Ritchie, ibid. 59, 2095 (1999).

${ }^{5}$ D. S. Kainth, D. Richards, H. P. Hughes, M. Y. Simmons, and D. A. Ritchie, J. Phys.: Condens. Matter 12, 439 (2000).

${ }^{6}$ H. Noh, S. Zelakiewicz, X. G. Feng, T. J. Gramila, L. N. Pfeiffer, and K. W. West, Phys. Rev. B 58, 12621 (1998).

${ }^{7}$ G. Senatore, F. Rapisarda, and S. Conti, Int. J. Mod. Phys. B 13, 479 (1999), and references therein.

${ }^{8}$ See for a general introduction, D. Pines and P. Nozières, The Theory of Quantum Liquids (Benjamin, New York, 1966), Vol. 1.

${ }^{9}$ K. S. Singwi, M. P. Tosi, R. H. Land, and A. Sjölander, Phys. Rev. 176, 589 (1968); K. S. Singwi and M. P. Tosi, Solid State Phys. 36, 177 (1981).

${ }^{10}$ C. Zhang and N. Tzoar, Phys. Rev. A 38, 5786 (1988).

${ }^{11}$ L. Zheng and A. H. MacDonald, Phys. Rev. B 49, 5522 (1994).

${ }^{12}$ D. Neilson, L. Świerkowski, J. Szymański, and L. Liu, Phys. Rev. Lett. 71, 4035 (1993).

${ }^{13}$ B. Dong and X. L. Lei, J. Phys.: Condens. Matter 10, 7535 (1998).

${ }^{14}$ L. Liu, L. Świerkowski, D. Neilson, and J. Szymański, Phys. Rev. B 53, 7923 (1996), and references therein.

${ }^{15}$ M. Alatalo, P. Pietiläinen, T. Chakraborty, and M. A. Salmi, Phys. Rev. B 49, 8277 (1994); M. Alatalo, M. A. Salmi, P. Pietiläinen, and T. Chakraborty, ibid. 52, 7845 (1995).

${ }^{16}$ J. T. Devreese, F. Brosens, and L. F. Lemmens, Phys. Rev. B 21, 1349 (1980); 21, 1363 (1980).

${ }^{17}$ K. Awa, H. Yasuhara, and T. Asahi, Phys. Rev. B 25, 3670 (1982); 25, 3687 (1982).

${ }^{18}$ P. K. Aravind, A. Holas, and K. S. Singwi, Phys. Rev. B 25, 561
(1982); A. Czachor, A. Holas, S. R. Sharma, and K. S. Singwi, ibid. 25, 2144 (1982).

${ }^{19}$ F. Green, D. Neilson, and J. Szymański, Phys. Rev. B 31, 2779 (1985); 31, 2796 (1985); 31, 5847 (1985); F. Green, D. Neilson, D. Pines, and J. Szymański, ibid. 35, 133 (1987).

${ }^{20}$ K. Takayanagi and E. Lipparini, Phys. Rev. B 54, 8122 (1996).

${ }^{21}$ A. Nakano and S. Ichimaru, Phys. Rev. B 39, 4930 (1989); 39, 4938 (1989).

${ }^{22}$ T. Hasegawa and M. Shimuzu, J. Phys. Soc. Jpn. 38, 965 (1975).

${ }^{23}$ A. Holas and S. Rahman, Phys. Rev. B 35, 2727 (1987).

${ }^{24}$ H. K. Schweng and H. M. Böhm, Phys. Rev. B 48, 2037 (1993).

${ }^{25}$ R. K. Moudgil, P. K. Ahluwalia, and K. N. Pathak, Phys. Rev. B 52, 11945 (1995).

${ }^{26}$ C. Bulutay and B. Tanatar, Europhys. Lett. 43, 572 (1998); B. Tanatar and C. Bulutay, Phys. Rev. B 59, 15019 (1999).

${ }^{27}$ C. F. Richardson and N. W. Ashcroft, Phys. Rev. B 50, 8170 (1994); N. P. Wang, J. Phys.: Condens. Matter 9, 6837 (1997); J. F. Dobson, M. J. Bünner, and E. K. U. Gross, Phys. Rev. Lett. 79, 1905 (1997).

${ }^{28}$ H. M. Böhm, S. Conti, and M. P. Tosi, J. Phys.: Condens. Matter 8, 781 (1996).

${ }^{29}$ The inhomogeneous free-electron response function is defined as $\chi_{0}\left(q, q^{\prime} ; \omega\right)=-(2 / \hbar) \int\left(d^{2} p / \hbar^{2}\right) \frac{f\left(p+\hbar q^{\prime} / 2\right)-f\left(p-\hbar q^{\prime} / 2\right)}{\omega-\mathbf{p} \cdot \mathbf{q} / m+i \eta}$,

where $f(p)$ is the Fermi distribution function. When $q^{\prime}=q$, the above expression reduces to the ordinary homogeneous response function.

${ }^{30}$ B. Tanatar and D. M. Ceperley, Phys. Rev. B 39, 5005 (1989).

${ }^{31}$ F. Rapisarda and G. Senatore, Aust. J. Phys. 49, 161 (1996).

${ }^{32}$ P. Phillips, Y. Wan, I. Martin, S. Knysh, and D. Dalidovich, Nature (London) 395, 253 (1998).

${ }^{33}$ S. Das Sarma and A. Madhukar, Phys. Rev. B 23, 805 (1981); G. E. Santoro and G. F. Giuliani, ibid. 37, 937 (1988); 37, 8443 (1988).

${ }^{34}$ G. J. Kalman, V. Valtchinov, and K. I. Golden, Phys. Rev. Lett. 82, 3124 (1999); see also for a review, K. I. Golden and G. J. Kalman, Phys. Plasmas 7, 4 (2000).

${ }^{35}$ J. Ortner, Phys. Rev. B 59, 9870 (1999).

${ }^{36}$ A. Holas and K. S. Singwi, Phys. Rev. B 40, 158 (1989); M. E. 
Bachlechner, H. M. Böhm, and A. Schinner, Phys. Lett. A 178, 186 (1993).

${ }^{37}$ K. N. Pathak and P. Vashishta, Phys. Rev. B 7, 4300 (1973).

${ }^{38}$ G. Mukhopadhyay, in Correlations in Electronic and Atomic Flu- ids, edited by P. Jena, R. Kalia, P. Vashishta, and M. P. Tosi (World Scientific, Singapore, 1990).

${ }^{39}$ P. Vashista and K. S. Singwi, Phys. Rev. B 6, 875 (1972).

${ }^{40}$ G. Vignale, Phys. Rev. B 38, 811 (1988). 